Article

\title{
Genomic Composition Permits Precise Analysis of a Novel High-Yielding Wheat Cultivar Bainong 207 and its Sister Lines
}

\author{
Chao $\mathrm{Ma}^{1+}$, Hao $\mathrm{Wu}^{2+}$, Sunish $\mathrm{K}$ Sehgal ${ }^{3+}$, Harsimardeep S Gill ${ }^{3}$, Xiubin Tian' ${ }^{1}$, Xingqi Ou${ }^{4}$, \\ Jianyong Chen ${ }^{2}$, Qidi Zhu' ${ }^{4}$, Huanhuan Li1 ${ }^{1}$ Zhenjie Dong1, Wenxuan Liu' ${ }^{*}$, Zengjun Qi $^{2}$ \\ ${ }^{1}$ The State Key Laboratory of Wheat and Maize Crop Science, College of Life Sciences, Henan Agricultural \\ University, Zhengzhou 450002, China; machao0813@hotmail.com (C.M.); XiubinTian2019@hotmail.com \\ (X.T.); lihuanhuanhappy@henau.edu.cn (H.L.); zhengjiedong@hotmail.com (Z.D.); \\ liuwenxuan@henau.edu.cn (W.L.) \\ 2 State Key Laboratory of Crop Genetics and Germplasm Enhancement, Nanjing Agricultural University, \\ Nanjing 210095, China; zjqi@njau.edu.cn (Z.Q.); 2018801213@njau.edu.cn (H.W.); 2017101106@njau.edu.cn \\ (J.C.) \\ ${ }^{3}$ Department of Agronomy, Horticulture and Plant Science, South Dakota State University, Brookings, SD \\ 57007, USA; sunish.sehgal@sdstate.edu (S.K.S.) ; harsimardeep.gill@sdstate.edu (H.G.) \\ ${ }^{4}$ School of Science and Technology, Henan Institute of Science and Technology, Xinxiang 453003, Henan \\ Province, China; ouyangxq@hist.edu.cn (X.O); zhuqidi@hist.edu.cn (Q.Z.) \\ * Correspondence: liuwenxuan@henau.edu.cn (W.L.); zjqi@njau.edu.cn (Z.Q.) \\ + These authors contributed equally to this work.
}

\begin{abstract}
Development and deployment of wheat varieties with high yields, wide adaptability, good quality, multiple-resistance to abiotic and biotic stresses, and efficient response to fertilizers have greatly contributed to global wheat sustainable production. The genomic composition of key commercial wheat variety can help understand the genetic basis underlying the development of new variety and permit increased breeding efficiency. In this study, we report the chromosomal and genomic compositions of BN207, presently the leading wheat variety in the southern region of Huang-Huai River Valley, the most important wheat producing area in China through an integrated analysis using fluorescent in situ hybridization (FISH) and wheat $15 \mathrm{~K} \mathrm{SNP}$ array. Our results showed that BN207 inherited 55.3\% and 40.7\% of its genome from its male parent BN64 and female parent ZM16, respectively, and generating 64 novel or recombined loci. Besides, we detected nine chromosomal variations in Bn207 and its parents and ten sister lines, and physically mapped two variations, the pericentric inversion of chromosome $6 \mathrm{~B}$, and large tandem repeat sequence block at the long arm of $5 \mathrm{~A}$, both had positive effects on agronomic traits, by integration of FISH and SNP loci recombination analyses. These results will provide a reference for breeding of high yield wheat varieties as BN207, and the application of founder parents BN64 and ZM16, which are being utilized frequently in wheat breeding programs in Henan Province and surrounding areas.
\end{abstract}

Keywords: Wheat variety BN207; Genome composition; FISH; SNP; Chromosomal variations

\section{Introduction}

Bread wheat (Triticum aestivum L., $2 \mathrm{n}=2 \mathrm{x}=14$, AABBDD) is an allohexaploid species of the genus Triticum that evolved through two independent natural hybridization events followed by chromosome doubling in the new hybrids. The first hybridization occurred between T. urartu Tumanian ex Gandilyan $(2 \mathrm{n}=2 \mathrm{x}=14, \mathrm{AA})$ and Aegilops speltoides Tausch $(2 \mathrm{n}=2 \mathrm{x}=14, \mathrm{SS})$ about 0.5 million years ago, forming a new tetraploid species T. turgidum $\mathrm{L}$. $(2 \mathrm{n}=2 \mathrm{x}=14$, AABB) [1]. The domesticated T. turgidum subsp. dicoccum then hybridized with another diploid species, Ae. tauschii Coss. ( $2 n=2 x=14, D D)$ about 7,000 to 12,000 years ago, producing a fertile hexaploid wheat seed ( $2 n$ $=6 \mathrm{x}=42, \mathrm{AABBDD})$ [2-7]. Although of relatively recent origin compared with its ancestors or other wild relatives, hexaploid bread wheat is currently the most widely grown crop, 218 million hm2 [8], 
and one of the most traded crops in the world. It is also the most favored staple food for humans and globally the leading source of vegetal protein [9-12]. Thus, sustainable production of wheat plays an important role in social progress and stability worldwide.

World wheat production has tripled since the green revolution in 1960s, and is expected to keep growing through the middle of the 21st century [13]. This increase was not achieved through expansion of wheat acreage but through increase crop yields per unit area and this has been a result of technological innovation and scientific crop management such as the improvement and deployment of novel varieties with better genetics, better seed storage, and germination ability, increased utilization of synthetic fertilizers and pesticides, and improved irrigation. Of these factors, development of novel wheat variety with objectives of high yields, wide adaptability, good quality, multiple-resistance to abiotic and biotic stresses, and efficient use of fertilizers has a major contribution [14-19].

The success of wheat breeding starts with the choice of suitable parents. A batch of several good varieties can often be derived from crosses involving some excellent founder parents. For example, Zhou 8425B was a wheat-rye spontaneous Robertsonian translocation (RT) 1BL.1RS stock derived from synthetic hexaploid Triticale $(2 n=6 x=42$, AABBRR), introduced from the International Maize and Wheat Improvement Center (CIMMYT) in 1970s [20,21]. Using it as a founder parent, as many as 100 varieties were developed in Henan Province and surrounding areas, of which up to 79 varieties, such as Zhoumai 16 and Aikang 58, were certificated for production at national or provincial level [22-24]. Xiaoyan 6, a wheat variety that is alleged derivative of a cross between wheat and Thinopyrum ponticum (Podp.) Barkw and D.R. Dewey $(2 \mathrm{n}=10 \mathrm{x}=70$, JSJSJSJSJJJJJJ) [25], was the largest planted wheat variety in its prime years in China. Using Xiaoyan 6 as a core parent more than 50 commercial varieties have been derived and released that occupied as large acreage, with cumulative extension area of 20 million $\mathrm{hm} 2$, and production increase of 15 million tones [26-29]. Italian wheat Funo and Abbondanza are founder parents to nearly 185 derivatives in China [26]. Thus, analysis of the genetic composition of a successful variety benefits our understanding of how to develop elite cultivar and improve key agronomic traits through breeding. This knowledge further helps in accelerating the breeding process. Thus, several attempts have been accomplished to elucidate the genetic basis of leading wheat cultivars and the founder parents using simple sequence repeat (SSR) markers [23, 30-33], high-density single nucleotide polymorphism (SNP) markers [34,35], or high-resolution fluorescent in situ hybridization (FISH) [36].

Bainong 207 (BN207) is a new wheat cultivar released and approved by the National Crop Variety Approval Committee of China in 2014. It has been the top-yielding variety with the largest annual acreage (1.35 million hm2) since 2016 in Henan Province and the southern region of HuangHuai River Valley of China, which is the most important wheat producing area in China [37]. The parents of BN207, Zhoumai 16 (ZM16) and Bainong 64 (BN64), also occupied large acreage in the region in their prime years [35,38-41]. In this study, we report the chromosomal and genomic composition of BN207, its parents and sister advanced lines through integrated analysis of highresolution FISH and wheat $15 \mathrm{~K} \mathrm{SNP}$ array to provide a reference for better utilization of BN207 and its founder parents, BN64 and ZM16, which widely used as parents in the southern region of HuangHuai River Valley of China.

\section{Materials and Methods}

\subsection{Plant Materials}

A total of thirteen varieties and advanced lines of wheat were used in this study. BN207 was derived from F8 derivatives of Zhoumai 16 (ZM16) as the female parent crossed with Bainong 64 (BN64), along with its ten sister advanced lines with different agronomic characteristics (Supplementary Table S1). All the materials are provided and maintained at the experiment station of Henan College of Science and Technology at Xinxiang, China.

\subsection{SNP Marker Assays}

Fresh leaves from twenty seedlings were collected and pooled for each plant material for DNA isolation. Genomic DNA was extracted using the CTAB (cetyltrimethylammonium Ammonium 
Bromide) method, and the integrity and concentration of DNA extracts were tested using agarose gel electrophoresis (1\%). The DNA of BN207, its parents and sister lines were hybridized on the wheat 15K SNP genotyping array containing 13,947 SNP markers designed by the Chinese Academy of Agricultural Sciences. The hybridization was completed by China Golden Marker Biotech Co. Ltd. (CGMB) (Beijing, China) following the Affymetrix Axiom 2.0 Assay Manual Workflow protocol.

\subsection{SNP Data Analysis and Genotype Map Construction}

The resulting SNP genotype data were used to analyze genomic composition in BN207, its parents and sister lines. If the genotype of BN207 at a particular locus was the same as that of a specific parent, then BN207 was considered to have inherited that locus from that particular parent. The loci present in BN207 but absent from both the parents are considered as novel loci, and loci limited to parental lines and absent in BN207 as deleted loci. Subsequently, the number of SNP loci contributed by each parent to the BN207 genome was calculated. The genomic contribution of a parent to BN207 was defined as the ratio of the number of SNP loci (allele) inherited from one parent as compared to another parent, based on the total number SNP loci polymorphic among the parents. SNP loci on different chromosomes were sorted from the short arm to the long arm, according to the sequence alignment between the flanking sequences of the SNP loci and the Chinese Spring RefSeq v.1.1 (IWGSC 2018). The genomic map was drawing based on SNP locations and sources at each chromosome. The physical position of each centromere was based on the International Wheat Genome Sequencing Consortium (IWGSC, 2018) [42]. Principal component analysis (PCA) for BN207 its parents and its sister lines were estimated in TASSEL v5.0 [43]. The first three eigenvectors were plotted as three principal components in CurlyWhirly v1.19.09.04 (https://ics.hutton.ac.uk/curlywhirly/) to observe clustering.

\subsection{Root-tip Chromosome Preparation and Fluorescent in situ Hybridization}

Chromosome spreads were prepared from root tip cells as described by Huang et al. (2018) [36]. The cytological observations were performed using a BX51 Olympus phase-contrast microscope (Olympus Corporation, Tokyo, Japan).

Dual-color none-denatured (ND)-FISH was performed using eight single-strand oligonucleotides as probes, including pAs1-1, pAs1-3, pAs1-4, pAs1-6, AFA-3, AFA-4, which were labeled with 6-carboxytetramethylrhodamine (TAMRA) generating red signals, pSc119.2-1 and (GAA)10 labeled with 6-carboxyfuorescein (FAM) generating green signals. All the oligonucleotides were synthesized at Sangon Biological Technology, Shanghai, China. The oligo probe of pSc119.2-1 was derived from the 118-bp repeat unit of plasmid clone pSc119.2, pAs1-1, pAs1-4 and pAs1-6 were derived from the $336 \mathrm{bp}$ repeat unit of plasmid clone pAs1, and AFA-3 and AFA- 4 were developed from a tandem sequence in GenBank accession AB003198.1 [44].

After hybridization, chromosomes were observed under an OLYMPUS BX51 (Olympus Corporation, Tokyo, Japan) fluorescence microscope and images were captured through a SPOT CCD (SPOT Cooled Color Digital Camera) and analyzed using Adobe Photoshop (v6.0) (Adobe, USA).

\subsection{Molecular Marker Analysis}

Sequences of PCR based primers for detection of pre-harvest sprouting resistance genes were deduced from the available reports [45-48], and synthesized by Sangon Biotech (Shanghai) Co., Ltd. China (Supplementary Table S2). PCR amplification was performed with $15 \mu \mathrm{L}$ of reaction mixture containing 7.5 $\mu \mathrm{L}$ of 2×EasyTaq PCR Supermix (TransGen Biotech, Beijiny, China), 0.5 pmol forward and reverse primers respectively, and $100 \mathrm{ng}$ of genomic DNA. PCR amplification was performed using F50SSR protocol followed Liu et al (2017) [49]. PCR products were resolved in 1.5\% agarose gels and visualized by ethidium bromide staining by a Tanon 2500 Gel Imaging System (Tanon Science \& Technology Co., Ltd., Shanghai, China). 


\section{Results}

\subsection{Yield Potential and Key Agronomic Traits of BN207}

BN207 is semi-dwarf wheat variety derived from the cross of cultivar ZM16 as female with BN64 as a male parent. ZM16 was characterized as a high yielding semi-dwarf variety with bigger spikes and larger grain size [41,50]; whereas, BN64 demonstrates comprehensive adult plant resistance to stripe rust and leaf rust, slow mildewing resistance, along with good quality and larger grain size [51,52]. Both ZM16 and BN64 are nationally certificated high yielding wheat varieties with an extensive area under cultivation and frequently deployed as founder parents in breeding programs in Henan and neighbor provinces. However, female parent ZM16 possesses some shortcomings including poor tolerance to low temperature in spring and pre-harvest sprouting, and average thousand-kernel weight (Supplementary Table S1). On the other hand, male parent BN64 has low tillering potential and weaker peduncle at maturity. Their derivative, BN207, successfully integrates the advantages and compensates the deficiencies of its parents, featured as high and stable yields, increased tolerance to low temperature in late spring, and pre-harvest sprouting, along with higher thousand-kernel weight.

Based on two years' regional trials in 37 locations and one year production trial at 14 locations in the southern region of Huang-Huai River Valley of China (conducted in collaboration by The Chinese National Agro-Tech Extension and Service Center, and Wheat Research Center in Henan Academy of Agricultural Sciences, Zhengzhou, China), BN207 showed an average yield of 7,542 to $8,761 \mathrm{~kg} / \mathrm{ha}$ from 2010 to 2013 , an increase of $3.8 \%$ to $7.0 \%$ over the commercial check variety ZM18 (Table 1). In 2014, the National Crop Variety Approval Committee of China approved BN207 for onfarm production and since then it has been the fastest-growing variety in Henan province. Ten sister lines of BN207 owing to different outstanding characteristics were selected from the same cross; however, none of them surpassed BN207 in yields and other agronomic characteristics (Supplementary Table S1).

Table 1. Yields and key agronomic characteristics of BN207

\begin{tabular}{cccccccc}
\hline Year & $\begin{array}{c}\text { Test } \\
\text { location }\end{array}$ & $\begin{array}{c}\text { Average } \\
\text { yield (kg/ } \\
\text { ha) }\end{array}$ & $\begin{array}{c}\text { Yield increase } \\
\text { over ZM18 } \\
\mathbf{( \% )}\end{array}$ & $\begin{array}{c}\text { Grain } \\
\text { number per } \\
\text { spike }\end{array}$ & $\begin{array}{c}\text { 1000- } \\
\text { grain } \\
\text { weight } \\
\mathbf{( g )}\end{array}$ & $\begin{array}{c}\text { Protein } \\
\text { content } \\
\mathbf{\%})\end{array}$ & Trial \\
\hline $2010-11$ & 20 & 8761.50 & $3.85^{* *}$ & 34.50 & 41.50 & 14.00 & Regional Trial \\
$2011-12$ & 17 & 7654.50 & $5.28^{* *}$ & 36.70 & 41.80 & 15.04 & $\begin{array}{c}\text { Regional Trial } \\
\text { 2012-13 }\end{array} 14$ \\
\hline
\end{tabular}

**: $1 \%$ level of significance

\subsection{Genomic Composition of BN207 and Contributions of Its Parents}

A total of 13,947 SNPs were assayed, however, 7,565 (54.2\%) good quality SNPs were selected for further analysis. These included 4,009 (52.9\%) monomorphic and 3,556 (47\%) polymorphic loci when compared among, BN64 and ZM16 the two parents of BN207. The maximum number of SNPs were found in sub-genome B, accounting for $41.3 \%$ of total SNPs and $51 \%$ of total polymorphic SNPs, followed by sub-genome A $(35.1 \%, 45.4 \%)$, and the least number of SNPs were from sub-genome D $(20.2 \%$ and $41.6 \%)$. These SNP loci were unevenly distributed at 21 different chromosomes, with average 360.2 SNP loci on each chromosome, ranging from maximum of 694 loci at chromosome 3B, followed by 528 on chromosome 2A, to 108 loci at chromosome 4D (Supplementary Table S3; Fig. 1). 


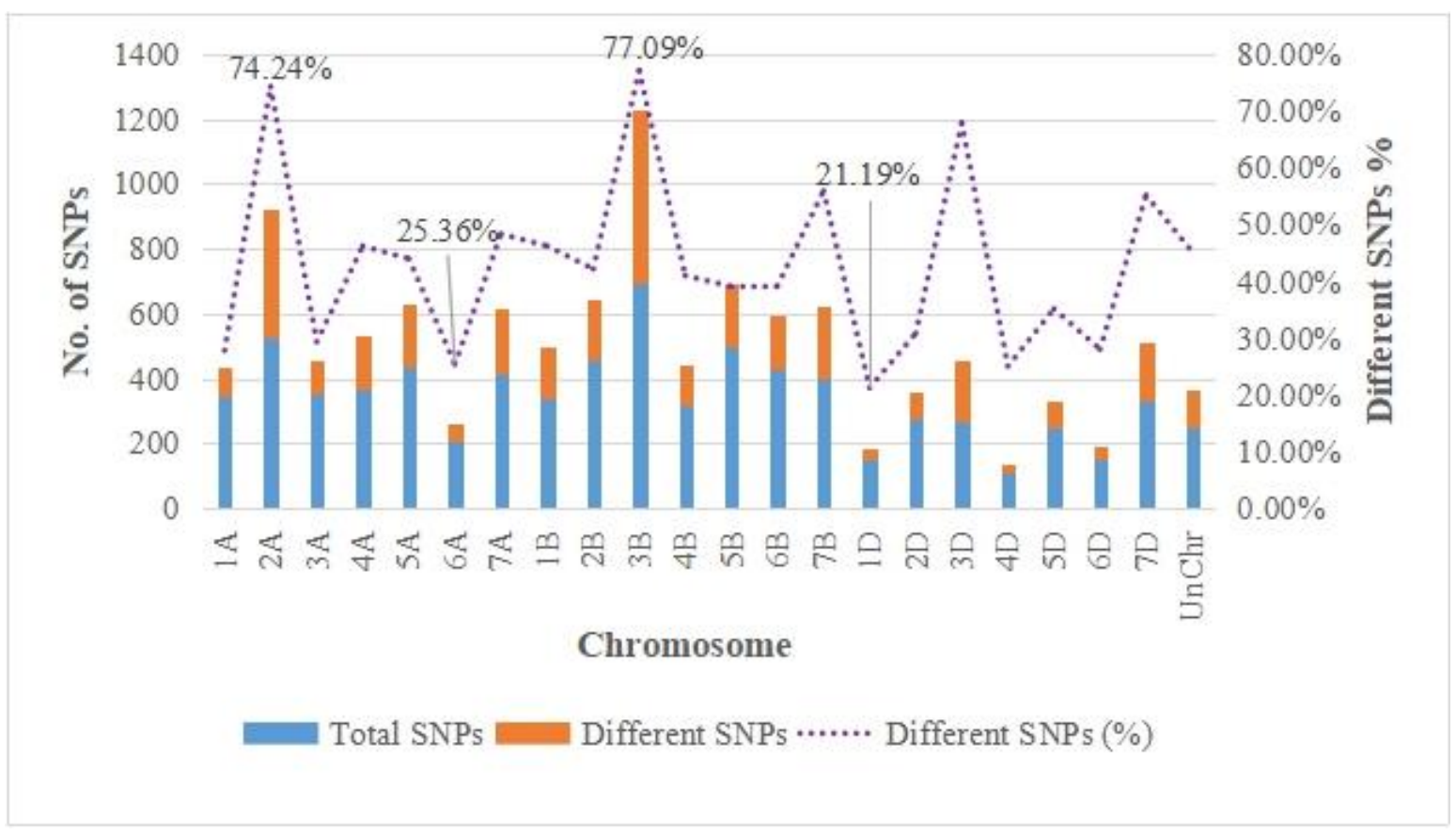

Figure 1. Chromosome distribution of SNP loci derived from parents BN64 and ZM16. UnChr: notassigned chromosome.

Genomic constitution of BN207 based on SNP loci showed that the male parent BN64 contributed 55.3\% (1,968 / 3,556 SNPs) of BN207 genome, whereas the female parent ZM16 contributed 40.7\% loci. The remaining 142 (3.2\%) SNP loci included 111 (3.1\%) heterozygous loci and $31(0.9 \%)$ novel or recombined loci present only in BN207 (Supplementary Table S4). In addition, 33 novel SNP loci were detected in BN207 from 4,009 SNP loci that were monomorphic among parents, increasing the total number of recombined loci to $64,0.85 \%$ of 7,565 SNPs analyzed. Parental contributions to BN207 were obviously different from sub-genomes, with an overwhelming majority of SNPs at B sub-genome derived from BN64, whereas relatively more loci at sub-genomes A and D from ZM16 (Fig. 2). About 149 polymorphic SNPs were from genes (exons) varied among BN207 and its sister lines and could be traced to one of the parents (Supplementary Table S5).

Analysis of the distribution of SNP loci in chromosomes of BN207 displayed significant differences of allele source (male or female parent)(Fig. 3). SNP loci in chromosome 3B, the short arm of chromosome 1B (1BS), the long arm of 2B (2BL), 2DL, 4AS, 5AS, 6A, 6B, 7AL and 7BS were predominantly derived from male parent $\mathrm{BN} 64$, whereas the majority alleles in chromosome $2 \mathrm{~A}, 3 \mathrm{~A}$, 3D, 4BS, 4D, 5BL, 6D, and 7D were originated from the maternal parent ZM16 (Fig. 3). Most of the heterozygous SNP loci were located at 5BL (34/111, 30.6\%), 4BL (20, 18.0\%) and 2BS (8, 7.2\%), while most of recombined loci were presented at $5 \mathrm{~B}(25 / 64,39.1 \%)$, followed by $3 \mathrm{D}(7,10.9 \%)$ in BN207 (Supplementary Table S4). 


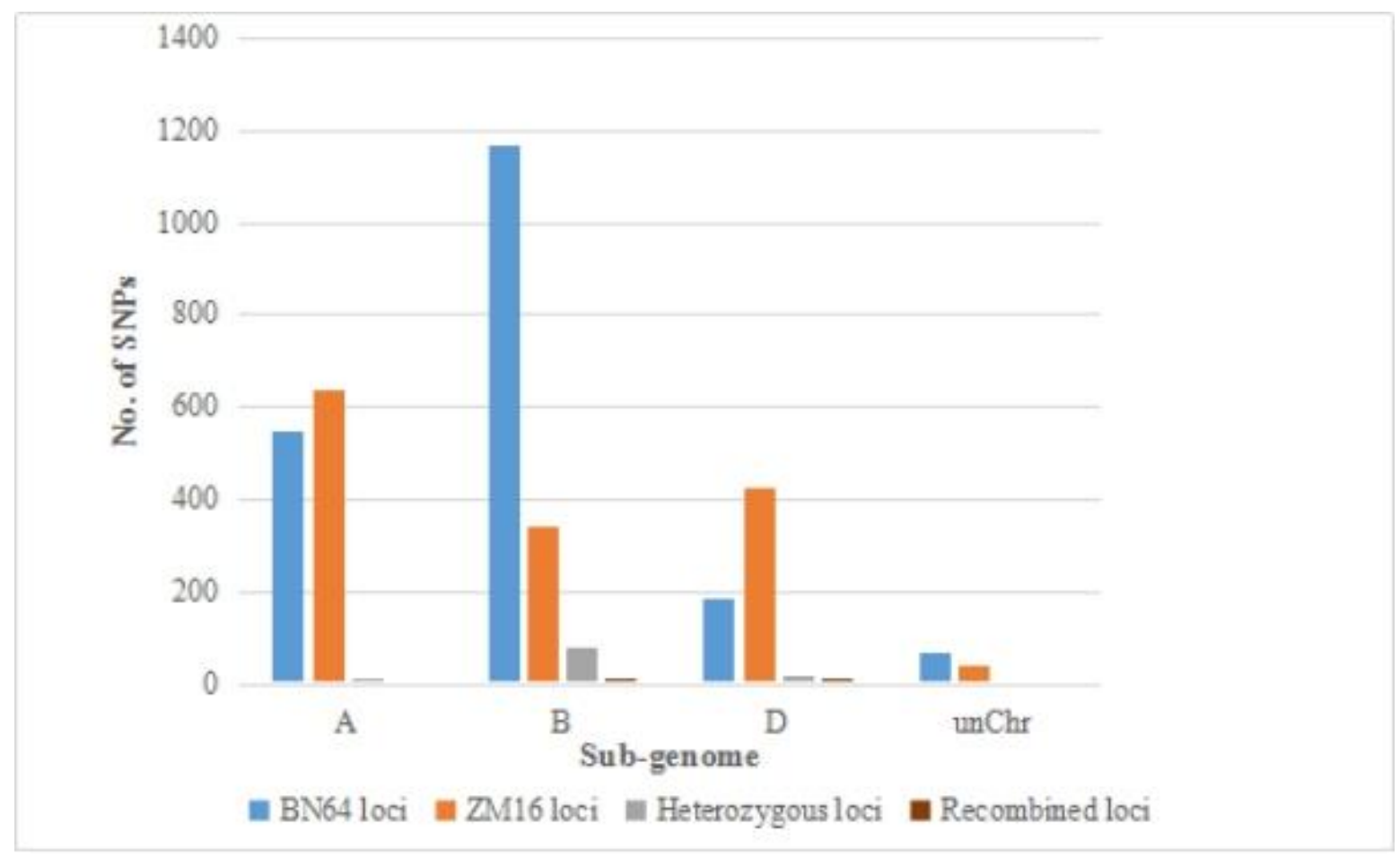

Figure 2. SNP locus derivative in wheat sub-genomes in BN207. Unchr: not-assigned chromosomes.

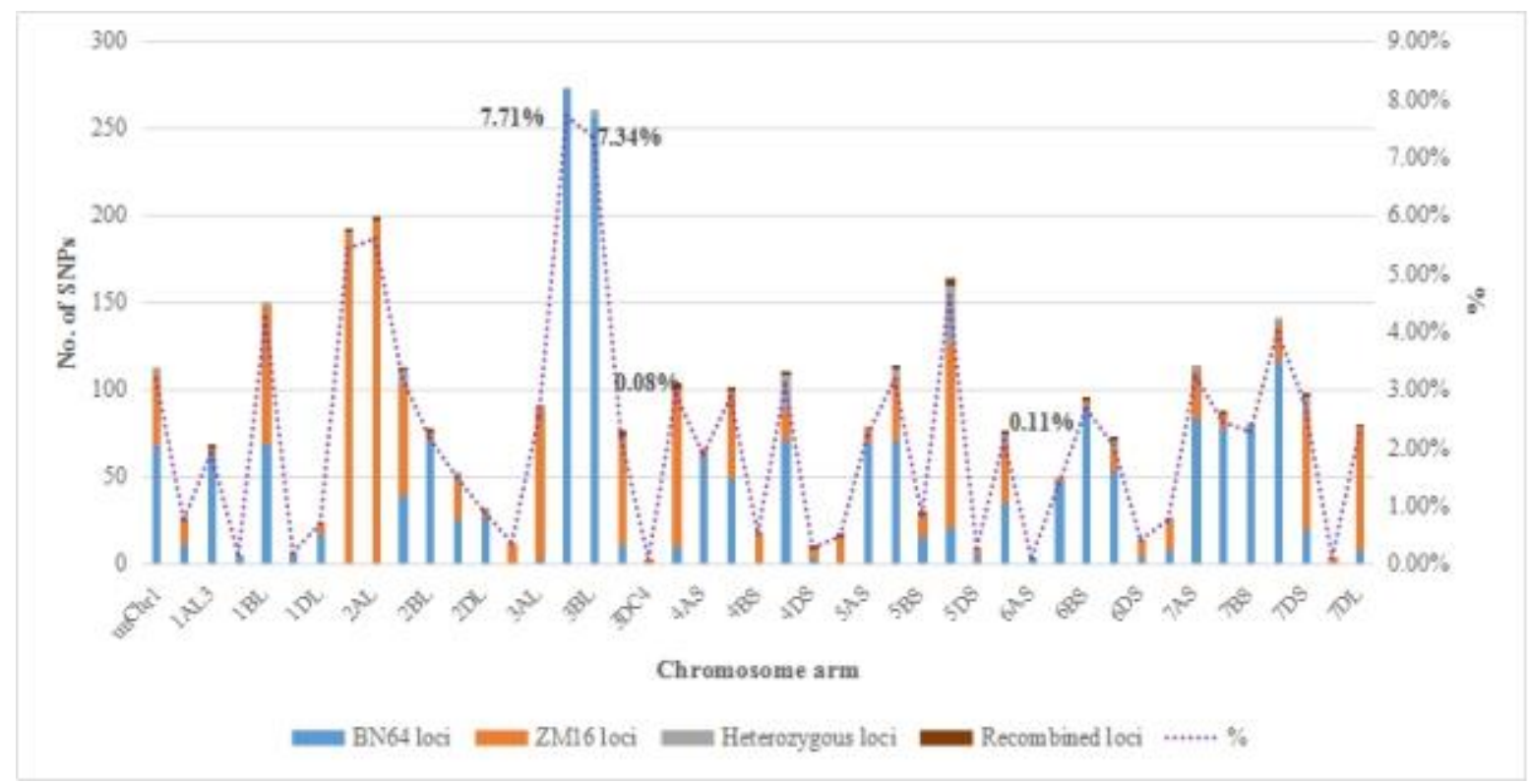

Figure 3. Chromosome-wise distribution and parental contribution of SNPs in BN207. Unchr: notassigned chromosomes.

\subsection{Comparison of SNP Loci in BN207 and Its Sister Lines}

Genotype map of SNP loci in BN207 and its ten sister lines were constructed based on allele and physical locations of SNP loci at different chromosomes (Fig. 4; Supplementary Table S6, S7). The map showed that the SNP loci inherited from parents were significantly varied among 11 derivatives. Most of the alleles/SNP at chromosome 1A, 4A, 6A, 3B were BN64-derived, whereas those at chromosome 2A, 5A, 1B, 6D and 7D were inherited from ZM16 in the majority of the advanced lines (Fig.4). 

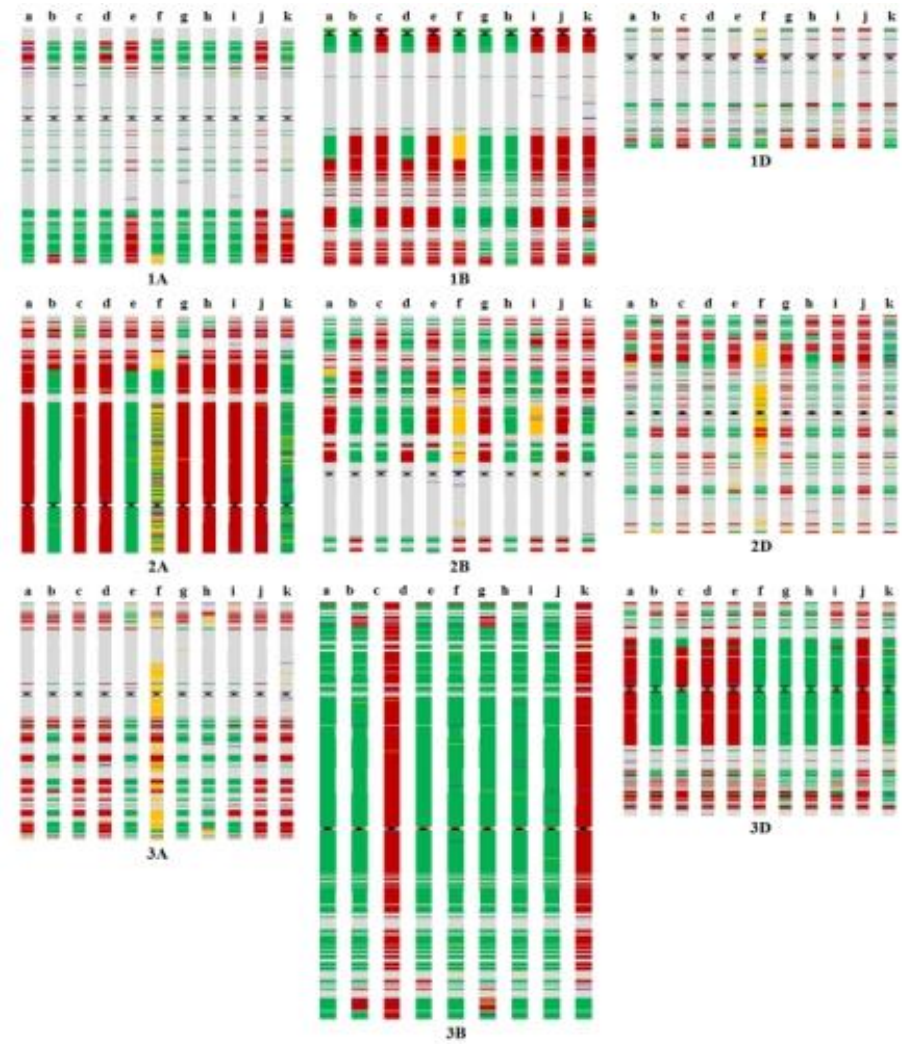

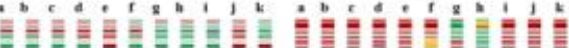
irifirition

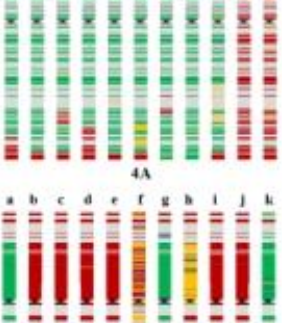
ioingion Eiti $D=D=D=D$

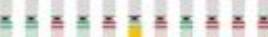
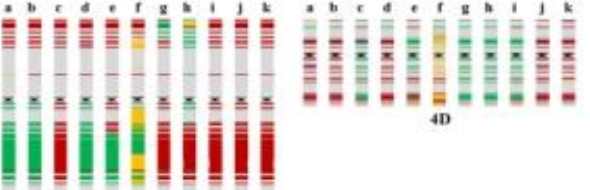

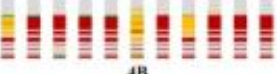

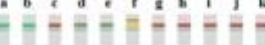

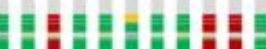

$\pm \pm= \pm= \pm=$
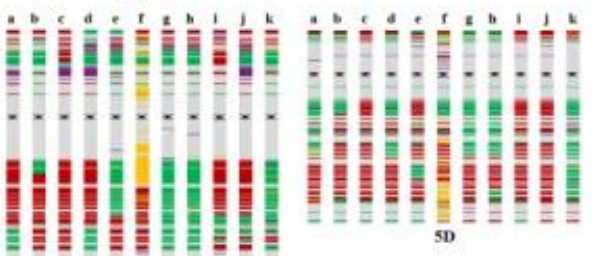

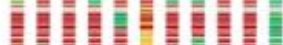
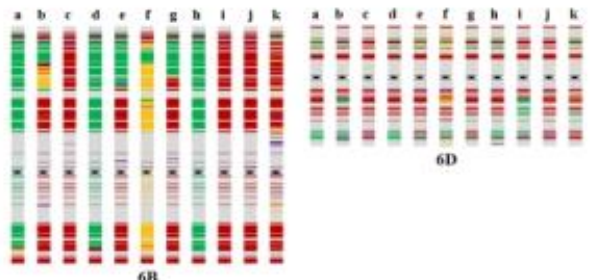

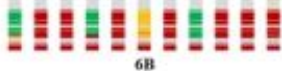
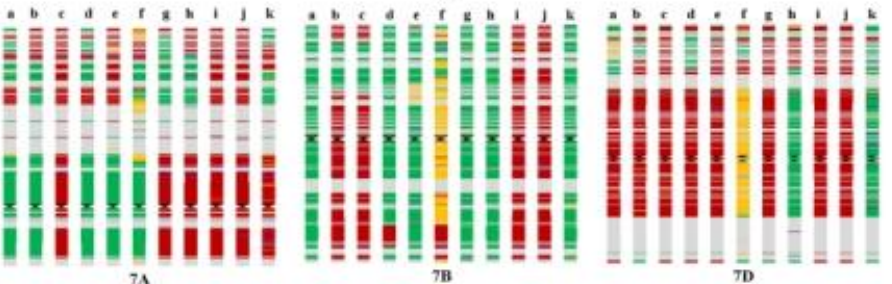

Figure 4. Genotype map of SNP loci in BN207, its parents and sister lines. Lane a to $\mathrm{k}$ represents BN207 (a), HY166 (b), GM1 (c), BH207 (d), BN69-83 (e), PJ11-15 (f), PJ11-52 (g), BN10-8 (h), BN12-40 (i), and BN14-818 (j), HY198 (k), respectively. Green represents SNP loci from BN64; red from ZM16; 
grey: monomorphic SNP loci; yellow: heterozygous loci; purple: novel or recombined SNP loci; black: centromeres. Chromosome length represents total number of loci at each chromosome.

The principle component analysis (PCA) showed that the first three components explained 64.6 $\%$ of variation among the parents and progeny. PCA distinguished BN207, its parents and sister lines into three clusters. The first cluster included ZM16 and three sister lines, BN14-818, BN12-40 and GM1, the second cluster comprised BN64, HY198, BN10-8, HY166, PJ11-52, and PJ11-15, the last one had BN207, and two sister lines, BH207 and BN69-38 (Fig. 5; Table S6). It also confirmed BN207, together with $\mathrm{BH} 207$, best-recombined different traits (SNP loci) from its two parent.

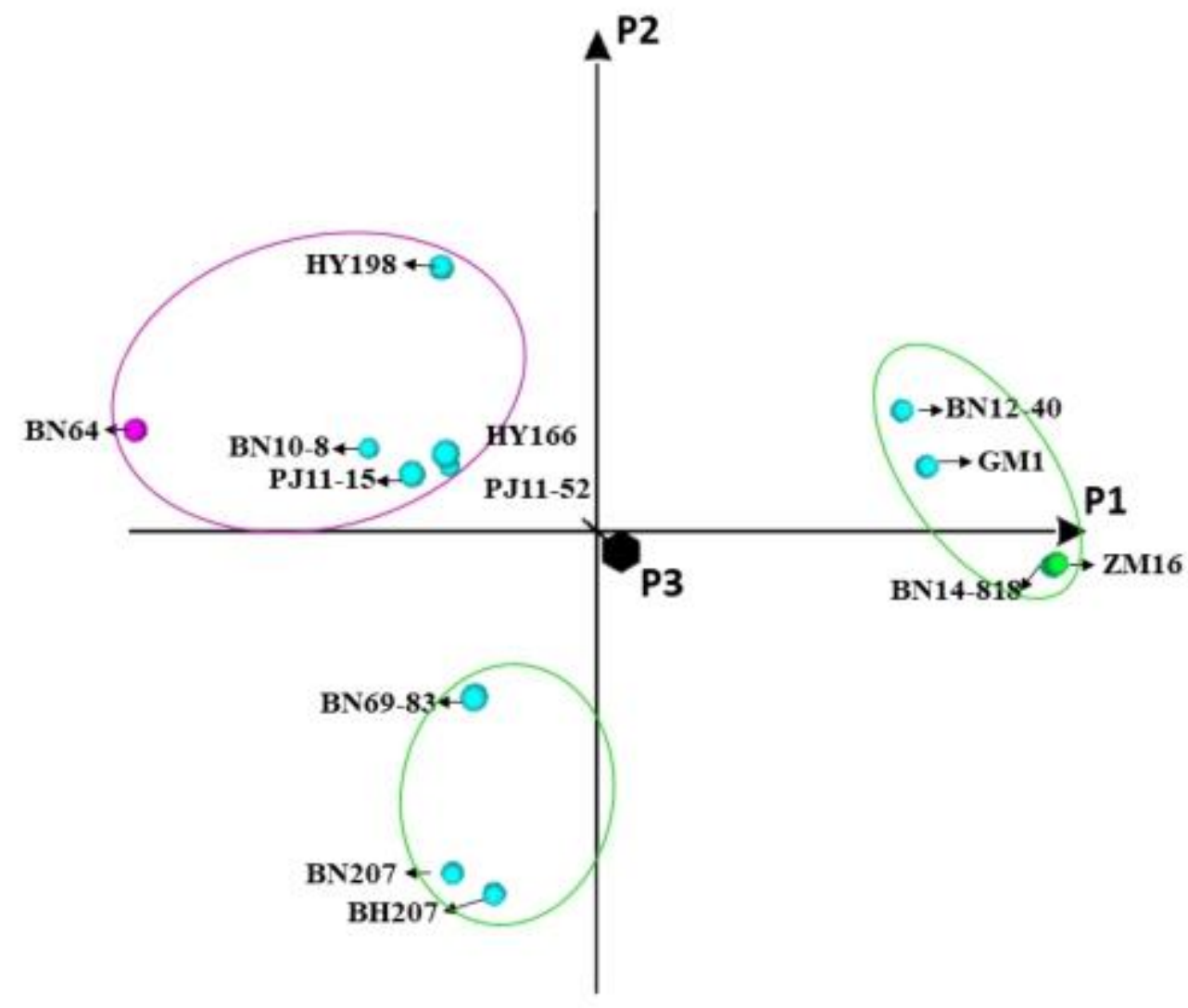

Figure 5. Genetic relationship among BN207, its parents and sister lines. Hierarchical clustering based principle component analysis (PCA).

Among the sister lines, BN14-818 was almost identical to the female parent ZM16, with as few as $3.55 \%$ of SNP loci different from ZM16. Of these 3.55\% SNPs, $1.66 \%$ SNPs were inherited from BN64, a majority (57.6\%) of them at chromosome 5B (Fig. 5; Fig. S1). Thus, this line could be used as a near-isogenic line of ZM16 to dissect the genetics underlying traits differing between BN14-818 and ZM16. Another line, BH207, which showed better drought tolerance compared to BN207 shared 89.8\% SNP loci with BN207. The remaining 10.2\% SNP differences between these lines may help in exploring genes related to yield advantage of BN207, and to drought-tolerance in BH207 (Fig. 5; Fig. S2). Besides, line PJ11-15 contained more than 32\% heterozygous SNP loci of the total, ranging from $0.00 \%$ to $75.00 \%$ across the 20 chromosomes except $3 \mathrm{~B}$, showing the advanced line is still genetically unstable (Fig. 4; Fig. S1).

Comparing BN207 with its parents and ten sister lines, only 0.75\% (57/7565) SNP loci were unique to BN207, of which 37 SNPs were heterozygous in BN207, whereas the remaining 20 SNP locations were deletions. These can probably be used as specific markers distinguishing BN207 from its parents and ten sister lines (Supplementary Table S8). 


\subsection{Chromosome Composition of BN207, Its Parents and Sister Lines}

BN207, its parents and ten sister lines selected for different agronomic characteristics, were used to perform Dual-color FISH. A total of 33 plants (1-4 plants each line) were analyzed for FISH. The results revealed nine chromosomal structural variations involving in the two parents BN64 and ZM16 and their derivative breeding lines. The pericentric inversion (PerInv) of chromosome 6B was detected in BN64, as previously identified by Huang et al. (2018) [36]. Another five structural variations include a Robertsonian translocation (RT) 1BL·1RS composed by the long arm 1B of wheat and the short arm of chromosome $1 \mathrm{R}$ from Secale cereale, a large AFA family tandem repeat sequence block (Trsb) represented by red color at the intermediate section of the 5AL (Figure 6), a green block displaying pSc119.2-1 and (GAA)10 repeats at the terminal region of 7BL, and one green signal at the terminal region of 7AS uniquely presented in ZM16. The three additional variations include a stronger green signal at the intercalary region of 3BL, and one larger green block at 4AL in BN64 ac compared toZM16, and a stronger red-colored AFA family repeats at chromosome 6BS in ZM16 when compared with BN64 (Fig.6).

Karyotype analysis indicated that BN207 inherited five chromosomal variations viz. 7A, 1B, 3B, perInv6B, and 1D, from BN64, and two variations, chromosome 4A, and 7B, from ZM16, and 5A and 6B involved recombination between the two parents. Chromosome 5A of BN207 looked like BN64 but with weaker green signal neighboring the centromere at $5 \mathrm{AL}$, and $6 \mathrm{~B}$ contained a pericentric inversion from $\mathrm{BN} 64$, and more red-colored AFA family repeats at terminal region at the short arm of 6B like ZM16. For the ten sister lines of BN207, five (HY198, GM1, BN14-818, BN12-40, and BN6938) had rearranged chromosome RT1BL.1RS from ZM16, four (GM1, BH207, BN12-40 and BN69-38) contained Trsb 5A from ZM16, and only two (BH207 and BN10-8) contained perInv 6B from BN64. Three lines (GM1, BN12-40 and BN69-38) had both structural variations, Trb 5A and RT1BL.1RS, from ZM16, and only BH207 had both perInv 6B and Trsb 5AL (Fig. 6; Fig. S3). The distribution of all variations in the eleven derivatives was shown in Fig. S4, which indicated most variations transmitted randomly with various ratios, but chromosomes $5 \mathrm{~A}, 6 \mathrm{~B}$ and $7 \mathrm{~B}$ involved more recombinations in the corresponding heterochromatin blocks of both parents. 


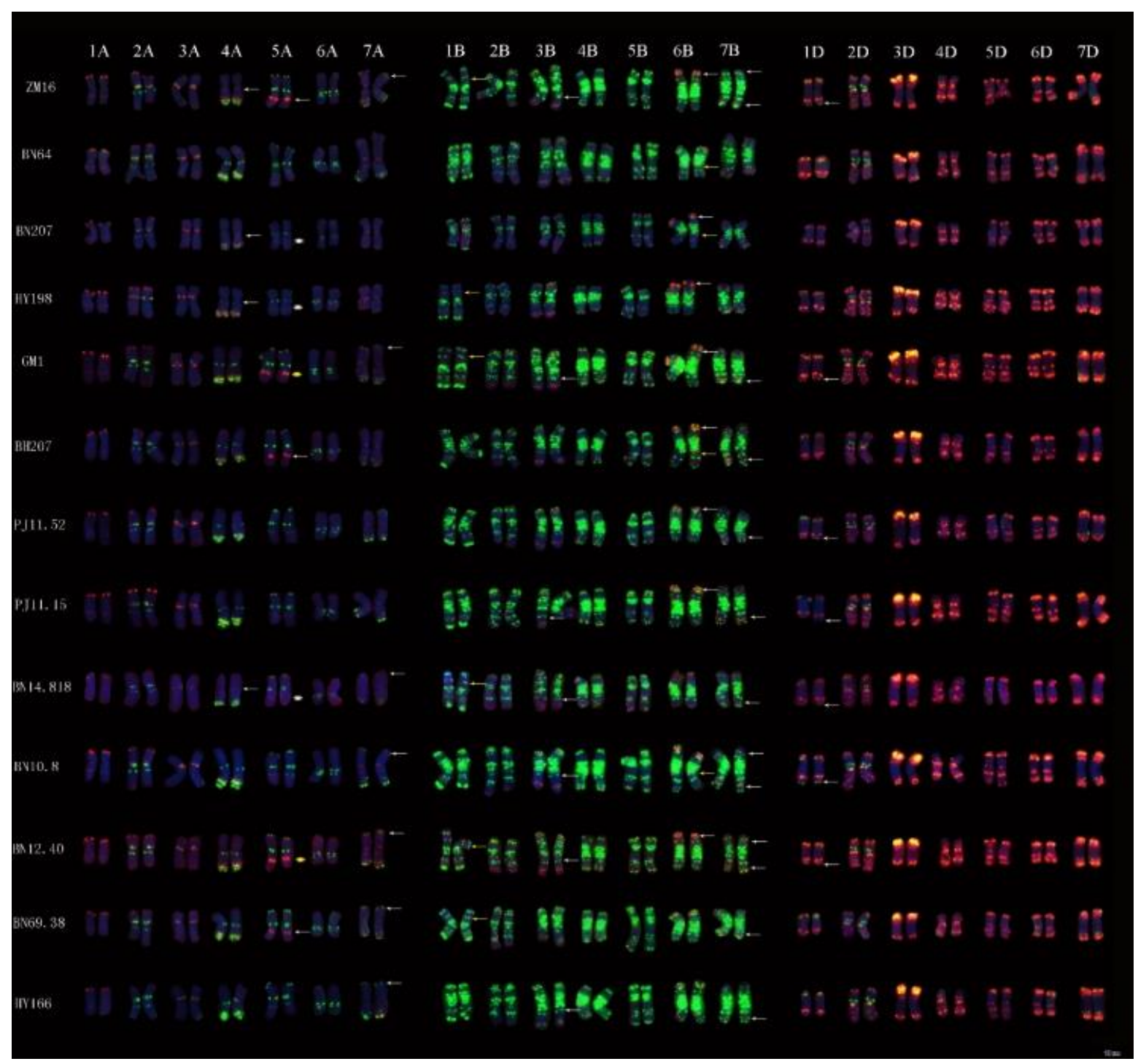

Figure 6. Karyotypes of BN207, its parents and sister lines after oligonucleotdie probe multiplex FISH. Probes pAs1-1, pAs1-3, pAs1-4, pAs1-6, AFA-3, and AFA-4 were labeled with 6carboxytetramethylrhodamine (TAMRA) and generating red signals; pSc119.2-1 and (GAA)10 labeled with 6-carboxyfuorescein (FAM), generating green signals. White arrows indicate polymorphism, yellow arrows indicate structural rearrangements, white and yellow asterisks indicate recombinations.

\subsection{Physical Mapping of Rearranged Chromosome Regions}

The physical position of SNPs on Chinese Spring reference sequence (IWGSC 2018) uncovers the allele at a locus, however, it fails to detect any chromosomal aberrations or variations identified in this study through FISH, including perInv 6B in BN64, Trsb 5A and RT1BL.1RS in ZM16. We integrated our FISH data with physical location of the SNP loci to identify the genomic region (size in $\mathrm{Mb}$ ) involved in chromosomal rearrangements. Analysis of alleles of SNP loci (parental sources) on chromosome 6B using three lines (BN207, BH207 and BN10-8) having perInv 6B showed that one recombination happened close to 6B centromere between SNP marker AX-109464405 at loci $42.5 \mathrm{Mb}$ and AX-110448396 at $50.1 \mathrm{Mb}$ at the short arm. The other recombination happened between AX109595550 at $475.5 \mathrm{Mb}$ and $\mathrm{AX}-111693296$ at $476.2 \mathrm{Mb}$ at the long arm of $6 \mathrm{~B}$. Thus, the pericentric inversion was mapped to a genomic region of $425.4 \mathrm{Mb}(50.1 \mathrm{Mb}$ at the short arm to $475.51 \mathrm{Mb}$ at the long arm) involving more than half of the length of the complete $6 \mathrm{~B}$ chromosome (Fig. 7; Table S9). Based on Wheat 15K SNP assay, this inverted region harbored a total of 261 SNP loci, including two 
functional SNP loci, AX-94384454 with positive additive effect on plant height at $115.95 \mathrm{Mb}$, and AX816177832 related to kernel length at $191.53 \mathrm{Mb}$ [53-55].

SNP loci analysis in four lines (GM1, BH207, 12-40 and 69-38) containing Trsb 5A indicated there were three blocks of non-recombination (recombination suppression) at Trsb 5A. The first block comprised complete short arm and the remaining two were located on the long arm, including a 312.9 $\mathrm{Mb}$ interval from the centromere $(109.1 \mathrm{Mb})$ to SNP loci AX-108726870 (422 Mb), and the other interval of $66.2 \mathrm{Mb}$ from SNP AX-110578543 (480.4 Mb) to AX-94589715 (546.5 Mb). Thus, the repeat sequence-block was predicted at the $66.1 \mathrm{Mb}$ interval was identified based on integrated analysis of FISH-displayed segment position and genomic positions of non-recombined SNP loci among the two parents (Fig. 7; Table S9).

Robertsonian translocation 1BL-1RS was identified in female parent ZM16, which was inherited from its male parent Zhoumai 8425B containing RT1BL-1RS different from Lovin series [23, 36]. Pairwise comparison of BN64-derived SNP loci and those from ZM16 in five advanced lines carrying RT1BL-1RS (HY198, GM1, 14-818, 12-40, and 69-38) showed recombination suppression between the short arms of $1 \mathrm{R}$ from rye and $1 \mathrm{~B}$ of wheat. At the long arm of chromosome $1 \mathrm{~B}$, no recombination was discovered at two intervals, the first one is $198.93 \mathrm{Mb}$ long from the centromere $(249.2 \mathrm{Mb})$ to SNP locus AX-110687238 at $448.13 \mathrm{Mb}$, and the second is $109.80 \mathrm{Mb}$ long, from SNP locus AX108725476 at $451.58 \mathrm{Mb}$ to AX-110091358 at $577.93 \mathrm{Mb}$ (Fig. 7; Table S9). No RT1BL-1RS chromosomes were found in BN207 and half of its ten sister lines, indicating this translocation once present in more than $70 \%$ of wheat varieties in Henan and neighboring provinces is gradually losing its domination to modern varieties of wheat [56].

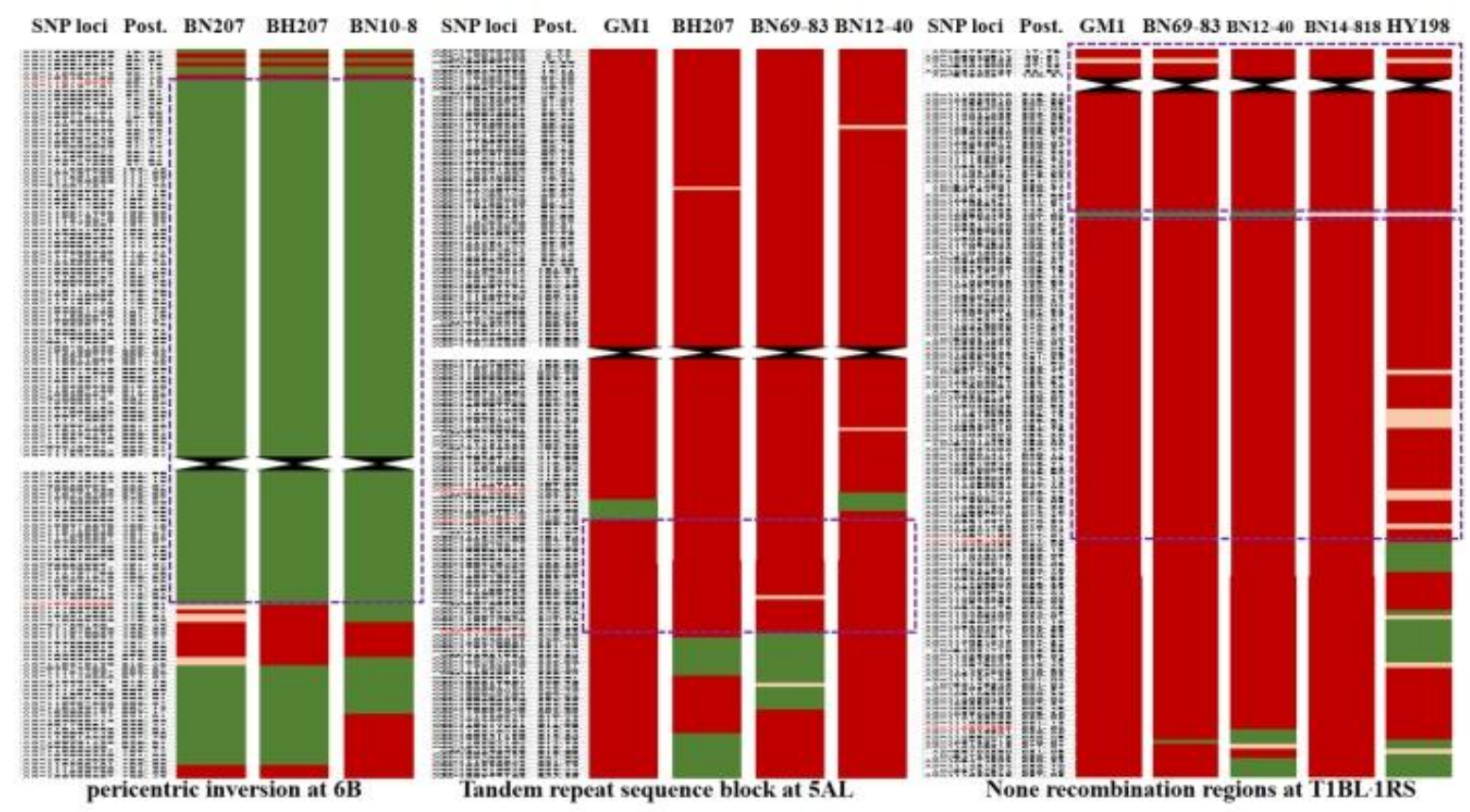

Figure 7. Physical mapping of chromosome structure variation in bi-parent. SNP loci from BN64 were presented in green color, and those from ZM16 in red color, deleted SNP loci which presented in parents but absent in BN207 or sister lines painted in yellow color, heterozygous loci in blue color, novel loci which were different from both parents painted in purple. The centromere of each chromosome is displayed in black color.

\subsection{Identification of the Resistance of Pre-harvest Sprouting in BN207}

BN207 was identified as a hard-white wheat with tolerance to pre-harvest sprouting, having lower ratios of both protrusion seeds and germinating seeds compared to the susceptible control Zhoumai 18 (ZM18) (Table 2). 
Table 2. Identification of resistance to pre-harvest sprouting of BN207 (at Xinxian, 2018)

\begin{tabular}{ccccccc}
\hline \multirow{2}{*}{ Variety } & \multicolumn{2}{c}{ None-germination rate (\%) } & \multicolumn{2}{c}{ Protrusion rate (\%) } & \multicolumn{2}{c}{ Germination rate (\%) } \\
\cline { 2 - 7 } & Average & Significance $^{*}$ & Average & Significance & Average & Significance \\
\hline BN207 & $69.58 \pm 2.72$ & $\mathrm{a}$ & $29.19 \pm 2.38$ & $\mathrm{~b}$ & $1.22 \pm 0.94$ & $\mathrm{c}$ \\
ZM18 (control) & $46.95 \pm 1.75$ & $\mathrm{~b}$ & $42.23 \pm 1.65$ & $\mathrm{a}$ & $10.79 \pm 1.50$ & $\mathrm{~b}$ \\
HY116 & $36.67 \pm 4.45$ & $\mathrm{c}$ & $44.15 \pm 5.60$ & $\mathrm{a}$ & $19.18 \pm 6.51$ & $\mathrm{a}$ \\
\hline
\end{tabular}

* Significant differences at $5 \%$ level of significance

Wheat 15K assay detected BN207 and its two parents, BN64 and ZM16, had two main effect QTL loci containing pre-harvest sprouting (PHS) resistance genes presented in Chinese wheat landraces. Of which, QTL1 on chromosome 3A is linked by SNP marker AX-111578083 located at $173.81 \mathrm{Mb}$, and QTL2 at chromosome 3D associated with AX-111624595 at $112.63 \mathrm{Mb}$. However, haplotype analysis showed BN207 and its parents were all same haplotypes, QTL1-HAP-G, and QTL2-HAP-T, which were unfavorable for PHS resistance [57].

To identify the genes involved in PHS resistance in BN207, we analyzed four PCR markers that are reported to be closely linked to PHS resistance genes (TaVp1B3, PM19-A1, Dorm, and TamyB10) (Supplementary Table S2). The results showed BN207 and its male parent BN64 displaying a $117 \mathrm{bp}$ fragment specific for PHS gene PM19-A1, and PHS gene TaVP1B3 specific marker of $569 \mathrm{bp}$ length, whereas no PHS gene-specific markers presented in female parent ZM16. Thus, BN207 contained at least two PHS resistance genes, PM19-A1 at $4 \mathrm{AL}$ and TaVP1B3 at 3BL, which were derived from its male parent BN64 (Fig. 8). PHS gene TaVP1B3 was further screened using thirty-four individual plants of BN207, and $24(70.59 \%)$ plants exhibited the $569 \mathrm{bp}$ fragment specific to the TaVP1B3. Thus, PHS resistance of BN207 could be further improved by selecting individuals having TaVP1B3 based on these markers.

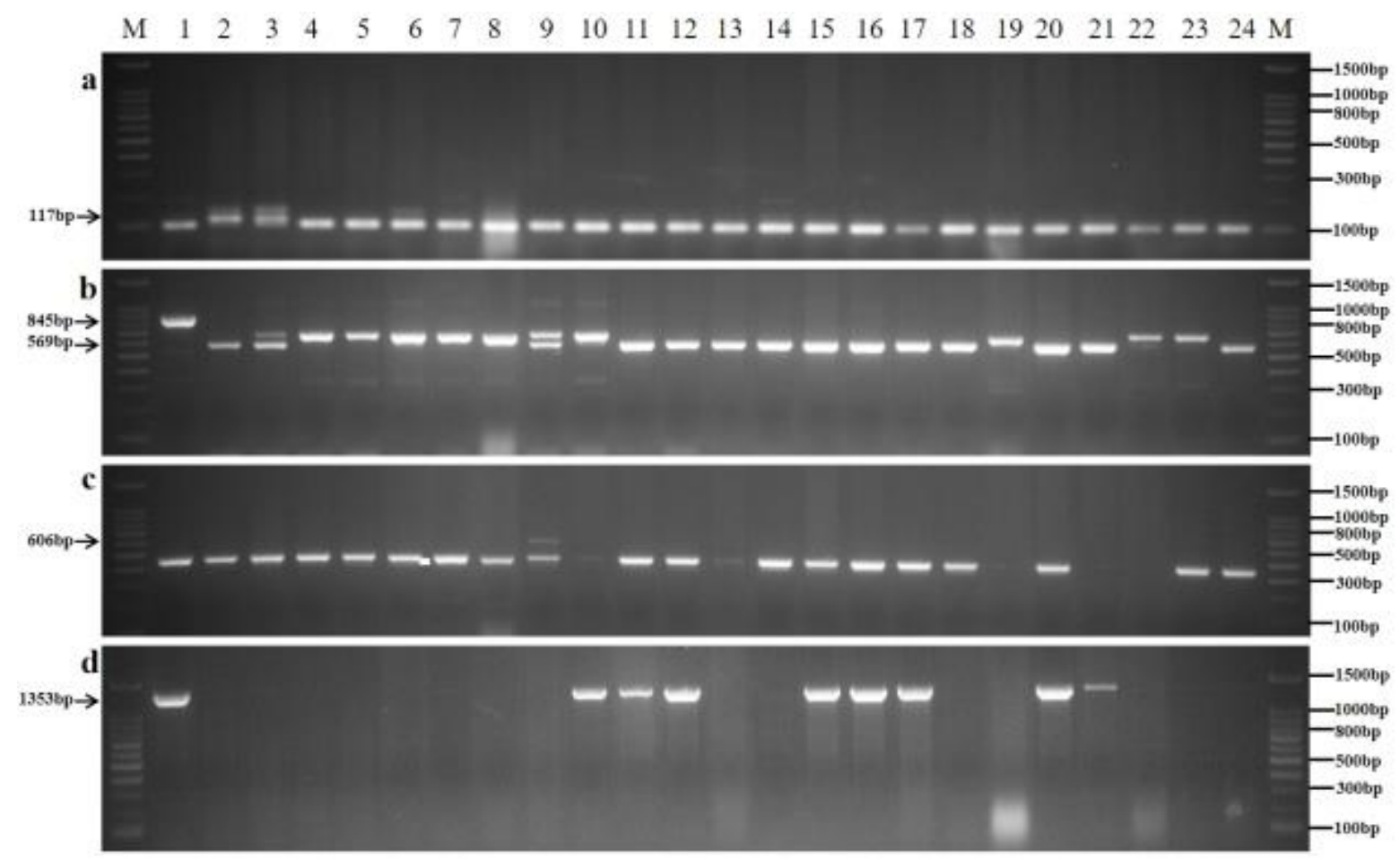

Figure 8. PCR patterns of BN207, its parents and other wheat varieties using PHS gene-specific premiers. M: 100 bp DNA loader; 1: Chinese Spring; 2: BN207; 3: BN64 ; 4: ZM16; 5 to 24: wheat varieties Zhoumai 16, Zhoumai 22, Ping'an 602, Ping'an 0518, Yangmai 5, Ningmai 9, Yangmai 13, Yangmai 14, Yangmai 16, Yangmai 17, Yangmai 23, Yangmai 158, NAU01, Nannong 0686, NAU617, Nannong 9918, Wangshuibai, Shengxuan 6, Yanzhan 4110, and Xinong 979. a to d: PHS gene-specific 
markers PM19-A1, TaVp1B3, Dorm-1, and TamyB10. Arrows indicate specific markers of PHS resistance genes.

\section{Discussion}

BN207 is a recently released wheat variety with the maximum acreage under cultivation demonstrating its broad adaptation and yield stability. Both parents of BN207, ZM16, and BN64, were commercial varieties grown on significant acres and are key founders of novel advanced lines and varieties from the southern region of Huang-Huai River Valley of China. Thus, dissection of genetic composition of BN207, and its parents will provide very important reference for wheat breeders. In this study we analyzed the genetic compositions of BN207, its parents BN64 and ZM16, and ten sister lines at both chromosome and genome level. We discovered that although BN64 contributed more SNP loci (55.3\%) than female parent ZM16 (40.7\%), the contributions to each chromosome were significantly different, of which SNPs on chromosome 1BS, 3BL, 6AS, 2AS, 4BS, 4DL almost derived from one parent, either BN64 or ZM16. Comparison of BN207 with ten sister lines revealed only 64 SNP loci unique to BN207, indicating the combination of different genes could be more important for the development of wheat varieties. Dissection of chromosome and genome composition of BN207 and its founder parents, BN64 and ZM16 will help breeders gain a better understanding of the genetic contribution of these varieties, benefit the rapid, accurate and consistent identification of the authenticity of cultivars, and improvement in breeding procedures, and protect the legal rights of breeders and enterprises.

Based on sequential genomic in situ hybridization (GISH)/FISH analysis, Huang et al (2018) [36] identified perInv 6B, originating from Italian wheat Funo, Abbondanza, Zhengyin 1 and Fan 6, was the most frequent type $(15.3 \%)$ in Chinese wheat varieties. However, the region of the pericentic inversion could not be mapped. In this study, we located the inversion at a $425.4 \mathrm{Mb}$ interval, from $50.1 \mathrm{Mb}$ at the short arm to $475.5 \mathrm{Mb}$ at the long arm of chromosome $6 \mathrm{~B}$ through the integration of FISH and SNP genotyping. The perInv 6B was identified inBN207 and its two sister lines (BH207 and 10-8) (Fig. S4). The second chromosomal variation Trsb 5A (derived ZM16) was present in close to half of the sister lines and was physically mapped in this study by integrating analysis of FISHdisplayed position and non-recombined SNP loci between two parents. Karyotype comparison revealed that five chromosomal variations in BN207 were derived from BN64, while another four were either from ZM16 or a result of recombination between the two parents, which was correspondent with the SNP genotyping results where BN207 was more similar to BN64. Chromosome variations either large structural rearrangements or polymorphism caused by large blocks of tandem repeats usually suppress the chromosome recombination, our study provides direct evidence about two such structural variations perInv6B and Trbs5AL.

Elite wheat variety BN207 its parents BN64 and ZM16 all cultivars grown widely-grown in Huang-Huai River Valley, China, suggest that perInv6B, Trsb 5A and RT1BL.1RS had likely a positive impact on agronomic characteristics. Despite the limited population size and SNP markers, we were able to pinpoint the precise location of chromosomal breakpoints, demonstrating that the cytological and SNP mapping information was an efficient way to identify chromosome structural rearrangements. The knowledge of chromosomal variations could be very important for future genetic mapping or genome re-sequencing in wheat. Further analysis using a large population permanent mapping population developed from BN64 and ZM16 combined with more SNP markers will provide precise information on such variations, which could also be helpful for mining the beneficial gene clusters involved in such genomic regions. In addition to the two structural changes, seven other chromosome structural polymorphisms also detected in this study. A further study on distribution these variations in modern elite cultivars and impacts to agronomic traits is underway at both chromosome and molecular level in our lab.

The wheat-rye RT1BL.1RS present in most of the Chinese cultivars can be traced to the founder wheat Lovrin series [30,36], which has played important role in wheat production both in China and the other regions of the world for more than 30 years. Whereas, wheat varieties derived from Zhou 8425B in Henan Province and surrounding area might have different 1RS origin. Zhou 8425B stock 
was developed in 1984 by Zhoukou Academy of Agriculture at Zhoukou, China, and its RT1BL.1RS derived from a synthetic hexaploid Triticale $(2 n=6 x=42$, AABBRR), introduced from CIMMYT in 1970s [20,21]. Presently, it is not clear whether the 1RS from this Triticale line is different from that of Lovrin series introduced from Romania and the Soviet Union in the 1970s [21]. ZM16 (female parent of BN207), is a nationally certified variety released in 2002 derived from the cross of Zhoumai 9 with Zhou 8425B, had a pair of rearranged RT1BL.1RS, whereas, BN207 and five of its sister lines showed absence of RT1BL.1RS. This indicates that this rearrangement is losing its dominance in modern varieties of wheat, at least in Henan Province and the southern region of Huang-Huai River Valley of China, mainly because its carries multiple disease resistance genes (Sr31, Lr26, and Yr9) and negative effect on grain quality [56].

In this study, BN207, an F8 progeny of ZM16 crossed with BN64, was screened for PHS resistance using a TaVP1B3 specific marker. We identified only $70.6 \%$ positive individual plants in the population suggesting that BN207 was heterogenous for TaVP1B3 and selection can further improve the resistance of BN207 to PHS.

\section{Conclusions}

Thus, our study, through the integrated use of genome-wide high-density SNPs and FISH revealed the genomic contribution of founder parents of the wheat cultivar BN207 and also identified several structural variations in BN207 and its sister lines. The characteristics of the genetic composition and structure of BN207 demonstrate the ability of precise evaluation of the genomic origin of cultivars by the integration of high throughput genotyping with FISH.

Supplementary Materials: The following are available online at www.mdpi.com/Agronomy/s1, Figure S1: SNP composition of each line, Figure S2: Similarity of sister lines to BN207, Figure S3: Individual chromosome variations, Figure S4: Distribution of chromosome variations, Table S1: title, Video S1: title. Table S1: Key characteristics of genotypes used in this study, Table S2: PHS resistance gene-specific markers used in this study, Table S3: SNPs assayed in BN64 and ZM16, Table S4: SNP loci derivatives and distribution in BN207, Table S5: Known genes inherited in BN207 and its sister lines from the parents ZM16 and BN64 and their function, Table S6: SNP data for cluster analysis, Table S7: SNP distribution at chromosomes of each sister line of BN207, Table S8: List of unique SNPs in BN207, Table S9: Physical map of chromosome structure rearrangements.

Author Contributions: W.L., Z.Q. and S.K.S. conceptualized the experiments and designed the methodology; C.M., X.T., Z.D. and H.L. performed the data curation and formal analysis; Z.D., H.W. and J.C. performed the FISH; X.O. and Q.Z supervised the field experiment; S.K.S. and H.J. perform cluster analysis and gene function annotation; W.L. Z.Q. and S.K.S. wrote the original manuscript. All authors commented and approved the final version.

Funding: This research was funded by the State Key Laboratory of Crop Genetics and Germplasm Enhancement at Nanjing Agricultural University, China (No. ZW2011002), the State Key Laboratory of Wheat and Maize Crop Science at Henan Agricultural University, China (No. 39990022), and the Scientific and Technological Research Major Project of Henan Province of China (No. 151100110700).

Acknowledgments: Thanks Mr. Beilin Wang for his support for drawing genetic maps.

Conflicts of Interest: The authors declare no conflict of interest.

\section{References}

1. Dvořak, J.; and Zhang, H. B. Variation in repeated nucleotide sequences sheds light on the phylogeny of the wheat B and G genomes. Proc Natl Acad Sci USA 1990, 87, 9640-9644.

2. Feldman, M.; Lupton, F.; Miller, T. E. In Wheats in Evolution of Crop Plants; Smartt, J. and Simmonds N.W. eds.; Longman Scientific: London, England, 1995, pp 184-192.

3. Feldman, M.; Levy, A. A. Allopolyploidy-a shaping force in the evolution of wheat genomes. Cytogenet Genome Res 2005,109, 250-258.

4. Huang, S.X.; Sirikachornkit, A., Su, X. J.; Faris, J.; Gill, B.; Haselkorn, R.; Gornicki P. Genes encoding plastid acetylCoA carboxylase and 3-phosphoglycerate kinase of the Triticum/Aegilops complex and the evolutionary history of polyploid wheat. PNAS 2002, 99, 8133-8138. 
5. Brenchley, R.; Spannagl, M.; Pfeifer, M.; Barker, G.L.; D'Amore, R.; Allen, A.M.; McKenzie, N.; Kramer, M.;

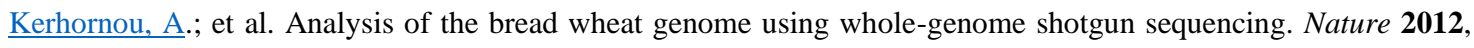
491, 705-710.

6. Liu, F.; Si, H.Q.; Wang, C.C.; Sun, G. L.; Zhou, E.; Chen, C.; Ma, C. Molecular evolution of Wcor15 gene enhanced our understanding of the origin of A, B and D genomes in Triticum aestivum. Sci Rep 2016, 6, 31706, doi: 10.1038/srep31706.

7. El Baidouri, M.; Murat, F.; Veyssiere, M.; Molinier, M.; Flores, R.; Burlot, L.; Alaux, M.; Quesneville, H.; Pont, C. and Salse, J. Reconciling the evolutionary origin of bread wheat (Triticum aestivum). New Phytol 2017, 213, 1477-1486.

8. Portmann, F.T.; Siebert, S.; and Döll, P. MIRCA2000-Global monthly irrigated and rainfed crop areas around the year 2000: A new high-resolution data set for agricultural and hydrological modeling, Global Biogeochem Cycles 2010, 24, GB1011, doi:10.1029/2008GB003435.

9. Lobell, D.B.; and Gourdji, S.M. The influence of climate change on global crop productivity. Plant Physiol 2012, 160, 1686-1697.

10. Shiferaw, B.; Smale,M.; Braun,H.J.; Duveiller, E.; Reynolds, M.; Geoffrey Muricho, G.Crops that feed the world 10. Past successes and future challenges to the role played by wheat in global food security. Food Sec 2013, 5, 291-317.

11. Shewry, P.R.; Hey, S.J. The contribution of wheat to human diet and health. Food Energy Secur 2015, 4, 178202.

12. Rasheed, A., Mujeeb-Kazi, A., Ogbonnaya, F. C., He, Z., \& Rajaram, S. Wheat genetic resources in the postgenomics era: promise and challenges. Annals of botany 2008, 121, 603-616, doi:10.1093/aob/mcx148.

13. Godfray, H.C.; Beddington, J.R.; Crute, I.R.; Haddad, L.; Lawrence D.; Muir J.F.; Pretty, J.; Robinson, S.;Thomas, S.M.; Toulmin, C. Food security: The challenge of feeding 9 billion people. Science 2010, 327, 812-818.

14. Feyerherm, A.M.; Paulsen, G.M.; and Sebaugh, J.L. Contribution of genetic improvement to recent wheat yield increases in the USA. Agronomy Journal 1984, 76, 985-990.

15. Brennan, J.P. Measuring the contribution of new varieties to increasing wheat yields. Review of Marketing and Agricultural Economics 1984, 52, 175-195.

16. Curtis, T.; Halford, N.G. Food security: the challenge of increasing wheat yield and the importance of not compromising food safety. Ann Appl Biol 2014, 164, 354-372.

17. Mazid, A., Keser, M., Amegbeto, K., Morgounov, A. Mzid A.; Keser, M.; Amegbeto, K.N.; Morgounov, A.; Bagci, A.; Peker, K.; Akin, M.; Kucukcongar, M.; Kan, M.; SemercI, A.; et al. Measuring the impact of agricultural research: The case of new wheat varieties in Turkey. Experimental Agriculture 2015, 51, 161-178.

18. Chenu, K.; Porter, J. R.; Martre, P.; Basso, B.; Chapman, S.C.; Ewert, F.; Bindi, M.; Asseng, S. Contribution of crop models to adaptation in wheat. Trends Plant Sci 2017, 22, 472-490.

19. Nalley, L., Dixon, B., Chaminuka, P., Naledzani, Z., Coale, M. J. The role of public wheat breeding in reducing food insecurity in South Africa. PLOS ONE 2018, 13: e0209598. doi: 10.1371/journal.pone.0209598

20. Zhao, X.L., Zheng, T.C., Xia, X.C.; He, Z.; Liu D.Q.; Yang, W.X.; Yin, G.H.; Li, Z.F. Molecular mapping of leaf rust resistance gene LrZH84 in Chinese wheat line Zhou 8425B. Theor Appl Genet 2008, 117, 1069-1075.

21. Tang, H.J.; Yin, G.H.; Xia, X.C.; Feng, J.J.; Qu, Y.Y; He, Z.H. Evaluation of molecular markers specific for 1BL·1RS translocation and characterization of 1RS chromosome in wheat varieties from different origins. Acta Agronomic Sinica 2009, 35, 2107-2115.

22. Yin, G.H.; Wang, J.W.; Wen, W.E.; He, Z.H.; Li, Z.F.; Wang, H.; Xia, X.C. Mapping of wheat stripe rust resistance gene YrZH84 with RGAP markers and its application. Acta Agron Sin 2009, 35, 1274-1281.

23. Xiao, Y.G.; Yin, G.H.; Li, H.H.; Yan, J.; Zheng, T.C.; Ji, W.Q.; He, Z.H. Genetic diversity and genome-wide association analysis of stripe rust resistance among the core wheat parent Zhou 8425B and its derivatives. Scientia Agricultura Sinica 2011, 44, 3919-3929.

24. Gao, F.; Wen, W.; Liu, J.; Rasheed, A.; Yin, G.; Xia, X.; Wu, X.; He, Z. Genome-wide linkage mapping of QTL for yield components, plant height and yield-related physiological traits in the Chinese wheat cross Zhou 8425B/Chinese Spring. Front Plant Sci 2015, 6, 1099.

25. Chen, Q.; Conner, R.; Laroche, A.; Thomas, J. Genome analysis of Thinopyrum intermedium and Thinopyrum ponticum using genomic in situ hybridization. Genome 1998, 41, 580-586.

26. Zhuang, Q. Chinese wheat improvement and pedigree analysis; China Agriculture Press: Beijing, China, 2003; pp 1-419, ISBN: 978-4-431-55674-9. 
27. Li, Z.S.; Li, B.; Tong, Y.P. The contribution of distant hybridization with decaploid Agropyron elongatium to wheat improvement in China. Genet Genomics 2008, 35, 451-456.

28. Li, Z.S.; Li; B.; Zheng, Q.; Li, H. In Advances in wheat genetics: from genome to field. Ogihara Y.; Takumi, S.; Handa, H. eds, Springer: Tokyo, Janpan, 2015; pp 377-385.

29. Qi, J.; Han, F.; Ma, S.; Zhang, L.; Yu X.; Chen Y.; Bi, X.; Shi, X.; Niu, N.; Zhang, G. Genetic dissection of wheat milestone parent Xiaoyan 6 and its derivatives. Journal of Northwest AEF University (Nat. Sci. ED.) 2015, 43, 45-53.

30. Xu, X.; Li, X.J.; Li X.Q.; Yang, X; Liu, W.; Gao, A.; Li, L.H. Inheritance of 1BL/1RS of founder parent Lovrin 10 in its progeny. J Triticeae Crops 2010, 30, 221-226.

31. Li, J.; Wan, H.; Yang W.; Wang, Q.; Zhu, X.; Hu, X.; Wei, H.; Tang, Y.;Li, C.; Peng, Z.; Zhou Y. Dissection of genetic components in the new high-yielding wheat cultivar Chuanmai 104. Scientia Agricultura Sinica 2014, 47, 2281-2291.

32. Li, X.;Hu, T.; Li, G.; Jiang, X.; Feng, S.; Dong, N.; Zhang, Z.; Ru, Z.; Huang, Y. Genetic analysis of broadgrown wheat cultivar Bainong AK58 and its sib lines. Acta Agronomica Sinica 2012, 38, 436-446.

33. Zou, S. K. et al. Genetic analysis of new wheat variety Zhoumai 23 and screening of specifc primers. Sci Agri Sin, 2015, 48, 3941-3951.

34. Jiang, P.; Zhang, P.; Zhang, X.; and Ma, H.X. Genetic contribution of Ningmai 9 wheat to its derivatives evaluated by using SNP markers. Int J of Genomics 2016, 2016, 3602986.

35. Li, C., Xu, W., Guo, R. et al. Molecular marker assisted breeding and genome composition analysis of Zhengmai 7698, an elite winter wheat cultivar. Sci Rep 2018, 8, 322.

36. Huang, X.; Zhu, M.; Zhuang, L.; Zhang, S.; Wang, J.; Chen, X.; Wang, D.; Chen, J.; Bao, Y.; Guo, J.; Zhang J4, Feng, Y.; Chu, C.; Du, P.; Qi, Z.; Wang, H.; Chen, P. Structural chromosome rearrangements and polymorphisms identified in Chinese wheat cultivars by 656 high-resolution multiplex oligonucleotide FISH. Theor Appl Genet 2018, 131, 1967-1986.

37. Wang, Y., He, H., Qiao, H., Ou, X. Study on appropriate combinations of sowing date and seeding amount for new wheat cultivar Bainong 207 in Henan province. J of Henan Institute of Science and Technology (Natural Science Edition) 2019, 47, 6-10.

38. Wang, Z.L.; Li, L.H.; He, Z.; Duan, X.; Zhou, Y.L.; Che, X.M.; Lillemo M.; Singh, R.P.; Wang, H.; Xia, X.C. Seedling and adult plant resistance to powdery mildew in Chinese bread wheat cultivars and lines. Plant Dis $89,2005,457-463$.

39. Wang, Z.; Wang, D.; He, Z.; Wang, H.; Chen, X.; Duan, X.; Zhou, Y.; Xia, X. QTL Mapping for slow mildewing resistance in Chinese wheat cultivar Bainong 64. Sci Agri Sin 2006, 39, 1956-1961.

40. Wang, L.; Yin, G.; Han, Y.; Huang, F.; Tang, J.; Yu, H.; Chen, L.; Yang G.; Li, X. Physiological and biochemical characteristics and grain filling mode of super-high-yield wheat Zhoumai16. Crop J 2010, 6, 8386.

41. Jin, H.; Wen, W.; Liu, J.; Zhai, S.; Zhang, Y.; Yan, X.; Liu, Z.; Xia, X.; He, Z. Genome-wide QTL mapping for wheat processing quality parameters in a Gaocheng 8901/Zhoumai 16 recombinant inbred line population. Front in Plant Sci 2016, 7, 1032. doi: 10.3389/fpls.2016.01032.

42. International Wheat Genome Sequencing Consortium (IWGSC). Shifting the limits in wheat research and breeding using a fully annotated reference genome. Science, 2018, 361, 6403.

43. Bradbury, P.; Zhang,Z.; Kroon, D.; Casstevens, T.; Ramdoss, Y.; Buckler, E. TASSEL: software for association mapping of complex traits in diverse samples. Bioinformatics 2007, 23, 2633-2635.

44. Du, P.; Zhuang, L.; Wang, Y.; Yuan, L.; Wang, Q.; Wang, D.; Dawadondup; Tan, Li.; Shen, J.; Xu, H.; Zhao, H.; Chu, C.; Qi, Z. Development of oligonucleotides and multiplex probes for quick and accurate identification of wheat and Thinopyrum bessarabicum chromosomes. Genome 2017, 60, 93-103.

45. Yang, Y.; Ma, Y.; Xu, Z.; Chen, X.; He, Z.; Yu, Z.; Wilkinson, M.; Jones, H.; Shewry, P.; Xia, L. Isolation and expression characterization of novel $V p-1$ genes in wheat varieties with distinct PHS tolerance and ABA responsiveness. J Exp Bot 2007, 58, 2863-2871.

46. Zhang, C. Development and validation of functional marker for resistance to pre-harvest sprouting. Ph. D dissertation, Chinese Academy of Agricultural Sciences, Beijing, China, 2008.

47. Himi, M.; Maekawa, M.; Miura, H.; Noda, K. Development of PCR markers for Tamyb10 related to R-1, red grain color gene in wheat. Theor and Appli Genet 2011, 22, 1561-1576.

48. Cao, X.; Zhang, H.; Jiang, H.; Wu, C.; Cao, J.; Zhu, Y.; Wang, S.; Chang, C.; Zhang, H.; Ma, C. Detection and validation of molecular marker PM19-A1 associated with pre-harvest sprouting resistance in 1015 wheat varieties. Journal of Triticeae Crops 2016, 36, 1283-1290. 
49. Liu, W.; Koo, D.; Xia, Q.; Li, C.; Bai, F.; Song, Y.; Friebe, B.; Gill B.S. Homoeologous recombination-based transfer and molecular cytogenetic mapping of powdery mildew-resistant gene Pm57 from Aegilops searsii into wheat. Theor Appl Genet 2017, 130, 841-848.

50. Zhai, S.; He, Z.; Wen, W.; Jin, H.; Liu, J.; Zhang, Y.; Liu, Z.; Xia, X. Genome-wide linkage mapping of flour color-related traits and polyphenol oxidase activity in common wheat. Theor Appl Genet 2016, 129: 377-394.

51. Lan, C.; Liang, S.; Wang, Z.; Yan J.; Zhang, Y.; Xia, X.; He Z. Quantitative trait loci mapping for adult-plant resistance to powdery mildew in Chinese wheat cultivar Bainong 64. Phytopathology 2009, 99, 1121-1126.

52. Ren, Y.; Li, Z.; He, Z.; Wu, L.; Bai, B.; Lan, C.; Wang, C.; Zhou, G.; Zhu, H.; Xia, X. QTL mapping of adult-plant resistances to stripe rust and leaf rust in Chinese wheat cultivar Bainong 64. Theor Appl Genet 2012, 125, 1253-1262.

53. Zanke, C.D.; Ling, J.; Plieske, J.; Kollers, S.; Ebmeyer, E.; Korzun, V.; Argillier, O.; Stiewe, G.; Hinze, M.; Neumann, K.; Gana, M.; Röder, M.S. Whole genome association mapping of plant height in winter wheat (Triticum aestivum L.). PLoS One 2014, 9(11): e113287. doi: 10.1371/journal.pone.0113287.

54. Zanke, C.; Ling, J.; Plieske, J.; Kollers, S.; Ebmeyer, E.; Korzun, V.; Argillier, O.; Stiewe, G.; Hinze, M.; Neumann, F. et al. Analysis of main effect QTL for thousand grain weight in European winter wheat (Triticum aestivum L.) by genome-wide association mapping. Front Plant Sci 2015, 6:644. doi: 10.3389/fpls.2015.00644.

55. Sun, C.; Zhang, F.; Yan, X.; Zhang, X.; Dong, Z.; Cui, D.; and Chen, F. Genome-wide association study for 13 agronomic traits reveals distribution of superior alleles in bread wheat from the Yellow and Huai Valley of China. Plant Biotechnol J 2017, 5, 953-969.

56. Tang, J.; Yin, G.; Gao, Y.; Wang, L.; Ji, W. Comprehensive analysis on agronomic traits and processing quality of core parent Zhou 8425B and its derivatives. J of Triticeae Crops 2015, 35, 777-784.

57. Zhou, Y.; Tang, H.; Cheng, M.; Dankwa, K.; Chen, Z.; Li, Z.; Gao, S.; Liu, Y.; Jiang, Q.;Lan, X.; Pu, Z.; Wei Y.; Zheng, Y.; Hickey, L.; Wang, J. Genome-Wide Association Study for Pre-harvest Sprouting Resistance in a Large Germplasm Collection of Chinese Wheat Landraces. Front Plant Sci 2017, 8:401. doi: 10.3389/fpls.2017.00401. 